International Journal of Pure and Applied Mathematics

Volume 86 No. 3 2013, 567-577

ISSN: 1311-8080 (printed version); ISSN: 1314-3395 (on-line version)

url: http://www.ijpam.eu

doi: http://dx.doi.org/10.12732/ijpam.v86i3.10

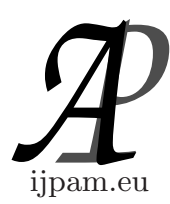

\title{
SOME LOCAL SUBSEMIHYPERGROUPS OF THE PARTIAL TRANSFORMATION SEMIGROUP ON A SET
}

\author{
Ruangvarin Intarawong Sararnrakskul \\ Department of Mathematics \\ Faculty of Science \\ Srinakharinwirot University \\ Bangkok, 10110, THAILAND
}

\begin{abstract}
In this paper, we give definitions of a local subset and a local subsemihypergroup of a semihypergroup. Moreover, we define a hyperoperation $\circ$ on the partial transformation semigroup $P(X)$ so that $(P(X), \circ)$ is a semihypergroup. Our aim is to characterize in form of an idempotent $\alpha$ of the semigroup $P(X)$ when the local subset $\alpha \circ G(X) \circ \alpha$ of $(P(X), \circ)$ become a local subsemihypergroup of $P(X)$.
\end{abstract}

AMS Subject Classification: 20M20, 20N20

Key Words: partial transformation semigroup, local subsemigroup, hypergroup, semihypergroup

\section{Introduction}

The notion of hyperstructure theory was introduced by F. Marty in 1934 at the 8th Congress of Scandinavian Mathematicians which plays an important role in the theory of algebraic hyperstructures. Up to present, many researchers have been studied in this area with classical algebraic structure, modern algebra and its application. The basic notations and results of the objects can be found in [2]. In 2003, Corsini and Leoreanu presented numerous application

Received: May 22, 2013

(c) 2013 Academic Publications, Ltd. url: www.acadpubl.eu 
of hyperstructures theory in their book [3]. These applications can be used in various areas such as graphs, combinatorial analysis, probability, geometry especially the theories of groups and semigroups.

First of all, we recall some basic definitions and examples of hypergroup theory from [2]. Let $H$ be a nonempty set and $P^{*}(H)$ the set of all nonempty subsets of $H$. A hyperoperation on $H$ is a function $\circ: H \times H \rightarrow P^{*}(H)$, the image of $(x, y) \in H \times H$ under $\circ$ is denoted by $x \circ y$ and called the hyperproduct of $x$ and $y$, and $(H, \circ)$ is called a hypergroupoid. For nonempty subsets $A$ and $B$ of $H$, let $A \circ B=\bigcup_{a \in A, b \in B} a \circ b, A \circ y=A \circ\{y\}$ and $x \circ B=\{x\} \circ B$ where $x, y \in H$.

A hypergroupoid $(H, \circ)$ is called a semihypergroup if the hyperoperation $\circ$ is associative, that is, $(x \circ y) \circ z=x \circ(y \circ z)$ for all $x, y, z \in H$.

A semihypergroup $(H, \circ)$ is called a hypergroup if $x \circ H=H \circ x=H$ for all $x \in H$.

A hypergroupoid $(H, \circ)$ is called commutative if $x \circ y=y \circ x$ for all $x, y \in H$.

If $(H, \circ)$ is a hypergroupoid [semihypergroup], then a nonempty subset $K$ of $H$ is called a subhypergroupoid [subsemihypergroupoid] if $K \circ K \subseteq K[K \circ K \subseteq$ $K]$, that is, $x \circ y \subseteq K$ for all $x, y \in K$.

One can see that semihypergroups are, in fact, generalization of hypergroups. Besides, hyperoperation o can be defined on a semigroup $S$ which makes $(S, \circ)$ a semihypergroup. Next, we would like to present some examples of semihypergroups and hypergroups.

Example 1.1. Let $H$ be a nonempty set and define $x \circ y=H$ for all $x, y \in H$. Then $(H, \circ)$ is a commutative hypergroup which is called the total hypergroup on $H$.

Example 1.2. Let $G$ be a group and for all $x, y \in G$ and define $x \circ y=$ $\langle x, y\rangle$, the subgroup of $G$ generated by $x$ and $y$. Then $(G, \circ)$ is a commutative hypergroup.

Example 1.3. Let $G$ be a group and $N$ a normal subgroup of $G$ and define $x \circ y=N x y$ for all $x, y \in G$. Then $(G, \circ)$ is a hypergroup (not necessary commutative).

Example 1.4. Let $S$ be a semigroup and $P$ be a nonempty subset of $S$ and define $x \circ y=x P y(=\{x t y \mid t \in P\})$ for all $x, y \in S$. Then $(S, \circ)$ is a semihypergroup. In particular, if $S$ is a group, then $(S, \circ)$ is a hypergroup. 
Proof. Let $x, y, z \in S$. Then

$$
\begin{aligned}
& (x \circ y) \circ z=(x P y) \circ z=\bigcup_{t \in x P y} t \circ z=\bigcup_{t \in x P y} t P z=(x P y) P z=x P y P z \text { and } \\
& x \circ(y \circ z)=x \circ(y P z)=\bigcup_{t \in y P z} x \circ t=\bigcup_{t \in y P z} x P t=x P(y P z)=x P y P z .
\end{aligned}
$$

If $S$ is a group or semigroup, then $S \circ x=\bigcup_{t \in S} t \circ x=\bigcup_{t \in S} t P x=S P x=S$ and $x \circ S=\bigcup_{t \in S} x \circ t=\bigcup_{t \in S} x P t=x P S=S$ which implies that $S$ is a hypergroup.

In [4], [6], [8] and [9], the authors use the word a "local subsemigroup" of a semigroup $S$ to mean a subsemigroup of $S$ of the form $e S e$ where $e$ is an idempotent of $S$. In 2008, [7] the authors were motivated by this definition and defined a "local subset" and a "local subsemigroup" of a semigroup $S$ in more general sense as follows : a local subset of a semigroup $S$ is a subset of $S$ of the form $e A e$ where $e$ is an idempotent of $S$ and $A$ is a subsemigroup of $S$. Note that a local subset of a semigroup $S$ need not be a subsemigroup of $S$. Then they were interested in finding a necessary and sufficient condition for an idempotent $e$ of $S$ which guarantees that $e A e$ becomes a subsemigroup of $S$ for a given subsemigroup $A$ of $S$. They called a local subset $e A e$ of a semigroup $S$ a local subsemigroup of $S$ if $e A e$ is a subsemigroup of $S$.

Denote by $E(S)$ the set of all idempotents of a semigroup $S$, that is,

$$
E(S)=\left\{x \in S \mid x^{2}=x\right\} .
$$

The cardinality of a set $X$ is denoted by $|X|$. The domain and range of a mapping $\alpha$ are denoted by $\operatorname{dom} \alpha$ and $\operatorname{ran} \alpha$, respectively, and the value of $\alpha$ at $x \in \operatorname{dom} \alpha$ is written by $x \alpha$. For $A \subseteq \operatorname{dom} \alpha, \alpha_{\left.\right|_{A}}$ denotes the restriction of $\alpha$ to $A$.

The partial transformation semigroup, the full transformation semigroup, the 1-1 partial transformation semigroup or the symmetric inverse semigroup and the symmetric group on a nonempty set $X$ are denoted $P(X), T(X)$, $I(X)$ and $G(X)$, respectively. In [7],the authors provided a neccessary and sufficient condition of $\alpha \in E(T(X))$, where $X$ is finite, so that $\alpha G(X) \alpha$ is a local subsemigroup of $T(X)$ as follows:

Theorem 1.5. (see [7]) Let $X$ be a finite nonempty set and $\alpha \in E(T(X))$. Then $\alpha G(X) \alpha$ is a local subsemigroup of $T(X)$ if and only if either

(i) $\alpha=1_{X}$, the identity mapping on $X$, or 
(ii) for every $a \in \operatorname{ran} \alpha,\left|a \alpha^{-1}\right| \geq|\operatorname{ran} \alpha|$.

In the second case, $\alpha G(X) \alpha=\alpha T(\operatorname{ran} \alpha) \cong T(\operatorname{ran} \alpha)$.

If $S$ is a semigroup and $\circ$ is a hyperoperation on $S$ making $(S, \circ)$ a semihypergroup, then we define a local subset and a local subsemihypergroup on $(S, \circ)$ in the similar way as follows : a local subset of a semihypergroup $(S, \circ)$ is a subset of $S$ of the form $e \circ A \circ e$ where $e$ is an idempotent of the semigroup $S$ and $A$ is a subsemihypergroup of $S$; moreover, if a local subset $e \circ A \circ e$ is a subsemihypergroup of $S$, then it is called a local subsemihypergroup of $S$.

In this paper, for a nonempty set $X$, we focus on the partial transformation semigroup $P(X)$ and its nonempty subset $I(X)$. Example 1.4, show that the semigroup $P(X)$ along with $I(X)$ form a semihypergroup under the hyperoperation $\circ$, namely,

$$
\alpha \circ \beta=\alpha I(X) \beta=\{\alpha \gamma \beta \mid \gamma \in I(X)\} \quad \text { for all } \alpha, \beta \in P(X) .
$$

Our aim is to investigate a characterization in term of an idempotent $\alpha$ of $P(X)$ to determine when the local subset $\alpha \circ G(X) \circ \alpha$ of $P(X)$ becomes a local subsemihypergroup of $P(X)$. Note that if $\alpha=0$ then $\alpha \circ G(X) \circ \alpha=$ $0 \circ G(X) \circ 0=\{0\}$ which is a trivial subsemihypergroup of $P(X)$. So, if $\alpha=0$ then $\alpha \circ G(X) \circ \alpha$ is a local subsemihypergroup of $P(X)$. From now on we will consider all idempotents $\alpha$ of $P(X)$ which is not 0 .

\section{Main Result}

First, we note that for any mapping $\alpha, \alpha$ can be written as

$$
\alpha=\left(\begin{array}{c}
x \alpha^{-1} \\
x
\end{array}\right)_{x \in \operatorname{ran} \alpha} .
$$

Moreover,

$$
\begin{aligned}
E(P(X)) & =\{\alpha \in P(x) \mid \operatorname{ran} \alpha \subseteq \operatorname{dom} \alpha \text { and } x \alpha=x \text { for all } x \in \operatorname{ran} \alpha\} \\
& =\left\{\alpha \in P(x) \mid \operatorname{ran} \alpha \subseteq \operatorname{dom} \alpha \text { and } \alpha_{\mid \operatorname{ran} \alpha}=1_{\operatorname{ran} \alpha}\right\}
\end{aligned}
$$

(see [1], page 12). To obtain our main result, the following series of lemmas is needed.

Lemma 2.1. Let $X$ be a nonempty set and $\alpha \in E(P(X)) \backslash\{0\}$. Then

$$
\alpha \circ G(X) \circ \alpha=\alpha I(X) \alpha .
$$


Proof. Clearly

$$
x \circ y \circ z=x \circ(y I(X) z)=\bigcup_{t \in y I(X) z} x \circ t=x I(X) y I(X) z
$$

for any $x, y, z \in P(X)$. It follows that

$$
\begin{aligned}
\alpha \circ G(X) \circ \alpha= & \bigcup_{\gamma \in G(X)} \alpha \circ \gamma \circ \alpha \\
& =\bigcup_{\gamma \in G(X)} \alpha I(X) \gamma I(X) \alpha \subseteq \alpha I(X) I(X) I(X) \alpha \subseteq \alpha I(X) \alpha
\end{aligned}
$$

and $\alpha I(X) \alpha=\alpha I(X) I(X) \alpha=\alpha I(X) 1_{X} I(X) \alpha=\alpha \circ 1_{X} \circ \alpha \subseteq \alpha \circ G(X) \circ \alpha$, thus $\alpha \circ G(X) \circ \alpha=\alpha I(X) \alpha$.

Next, Lemma 2.2. is provided to show that $\alpha P(\operatorname{ran} \alpha)$ and $P(\operatorname{ran} \alpha)$ are subsemihypergroups of $P(X)$ that is a Lemma 2.3.

Lemma 2.2. Let $X$ be a nonempty set and $\alpha \in E(P(X)) \backslash\{0\}$. If $\beta, \gamma \in P(\operatorname{ran} \alpha)$, then $\alpha \beta I(X) \alpha \gamma=\alpha \beta I(X) \gamma$.

Proof. Let $\beta, \gamma \in P(\operatorname{ran} \alpha)$ and $f \in I(X)$. Consider $f \gamma$. Since $\operatorname{ran} f \cap$ $\operatorname{dom} \gamma \subseteq \operatorname{ran} \alpha$ and $\alpha_{\left.\right|_{\operatorname{ran} \alpha}}=1_{\operatorname{ran} \alpha}$, it follows that $y \alpha=y$ for any $y \in$ $\operatorname{ran} f \cap \operatorname{dom} \gamma$. Thus, for any $x \in \operatorname{dom} f \gamma, x f \gamma=(x f) \gamma=((x f) \alpha) \gamma=x f \alpha \gamma$. Moreover,

$$
\begin{aligned}
\operatorname{dom}(f \alpha \gamma) & =[\operatorname{ran} f \cap \operatorname{dom}(\alpha \gamma)] f^{-1} \\
& =\left[(\operatorname{ran} f) \cap(\operatorname{ran} \alpha \cap \operatorname{dom} \gamma) \alpha^{-1}\right] f^{-1} \\
& =\left[(\operatorname{ran} f) \cap(\operatorname{dom} \gamma) \alpha^{-1}\right] f^{-1} \quad(\because \operatorname{dom} \gamma \subseteq \operatorname{ran} \alpha) \\
& =[\operatorname{ran} f \cap \operatorname{dom} \gamma] f^{-1} \quad\left(\because \alpha_{\mid \operatorname{ran} \alpha}=1_{\operatorname{ran} \alpha}\right) \\
& =\operatorname{dom} f \gamma .
\end{aligned}
$$

This proves that $f \gamma=f \alpha \gamma$ leading to $\alpha \beta f \gamma=\alpha \beta f \alpha \gamma$ for any $f \in I(X)$. Consequently, $\alpha \beta I(X) \gamma=\alpha \beta I(X) \alpha \gamma$.

Lemma 2.3. Let $X$ be a nonempty set and $\alpha \in E(P(X)) \backslash\{0\}$. Then $\alpha P(\operatorname{ran} \alpha)$ and $P(\operatorname{ran} \alpha)$ are subsemihypergroups of $P(X)$ and

$$
\alpha P(\operatorname{ran} \alpha) \cong P(\operatorname{ran} \alpha)
$$

under good isomorphism of semihypergroups. 
Proof. If $\beta \in P(X)$, then $\operatorname{ran}(\beta \alpha) \subseteq \operatorname{ran} \alpha$, so $\alpha \beta \alpha=\alpha\left((\beta \alpha)_{\left.\right|_{\operatorname{ran} \alpha}}\right) \in$ $\alpha P(\operatorname{ran} \alpha)$. Thus $\alpha P(X) \alpha \subseteq \alpha P(\operatorname{ran} \alpha)$. It follows that

$$
\begin{aligned}
(\alpha P(\operatorname{ran} \alpha)) \circ(\alpha P(\operatorname{ran} \alpha)) & =\bigcup_{x, y \in \alpha P(\operatorname{ran} \alpha)} x \circ y \\
& =\bigcup_{x, y \in \alpha P(\operatorname{ran} \alpha)} x I(X) y \\
& =\alpha P(\operatorname{ran} \alpha) I(X) \alpha P(\operatorname{ran} \alpha) \\
& =\alpha(P(\operatorname{ran} \alpha) I(X)) \alpha P(\operatorname{ran} \alpha) \\
& \subseteq \alpha P(X) \alpha P(\operatorname{ran} \alpha), \\
& \subseteq \alpha P(\operatorname{ran} \alpha) P(\operatorname{ran} \alpha) \\
& \subseteq \alpha(P(\operatorname{ran} \alpha),
\end{aligned}
$$

so $\alpha P(\operatorname{ran} \alpha)$ is a subsemihypergroup of $P(X)$ and

$$
\begin{aligned}
P(\operatorname{ran} \alpha) \circ P(\operatorname{ran} \alpha) & =\bigcup_{x, y \in P(\operatorname{ran} \alpha)} x \circ y \\
& =\bigcup_{x, y \in P(\operatorname{ran} \alpha)} x I(X) y \\
& =P(\operatorname{ran} \alpha) I(X) P(\operatorname{ran} \alpha) \\
& \subseteq P(\operatorname{ran} \alpha) P(X) P(\operatorname{ran} \alpha) \\
& \subseteq P(\operatorname{ran} \alpha),
\end{aligned}
$$

thus $P(\operatorname{ran} \alpha)$ is a subsemihypergroup of $P(X)$.

Next, we define $\varphi: P(\operatorname{ran} \alpha) \rightarrow \alpha P(\operatorname{ran} \alpha)$ by $\varphi(\beta)=\alpha \beta$ for all $\beta \in P(\operatorname{ran} \alpha)$. Then $\varphi$ is onto and for $\beta, \gamma \in P(\operatorname{ran} \alpha)$,

$$
\begin{aligned}
\varphi(\beta \circ \gamma) & =\{\varphi(x) \mid x \in \beta \circ \gamma\} \\
& =\{\alpha x \mid x \in \beta I(X) \gamma\} \\
& =\{\alpha \beta \lambda \gamma \mid \lambda \in I(X)\} \\
& =\alpha \beta I(X) \gamma \\
& =\alpha \beta I(X) \alpha \gamma \quad \text { by Lemma } 2.2 \\
& =(\alpha \beta) \circ(\alpha \gamma) \\
& =\varphi(\beta) \circ \varphi(\gamma) .
\end{aligned}
$$

Therefore $\varphi$ is a good homomorphism. 
Next, assume that $\varphi(\beta)=\varphi(\gamma)$. Then $\alpha \beta=\alpha \gamma$. Let $x \in \operatorname{dom} \beta$. Then $x \in \operatorname{ran} \alpha$, so $x \alpha=x$. Thus

$$
x \beta=(x \alpha) \beta=x(\alpha \beta)=x(\alpha \gamma)=(x \alpha) \gamma=x \gamma,
$$

so $x \in \operatorname{dom} \gamma$. This shows that $\operatorname{dom} \beta \subseteq \operatorname{dom} \gamma$ and $x \beta=x \gamma$ for all $x \in \operatorname{dom} \beta$. It can be shown similary that $\operatorname{dom} \gamma \subseteq \operatorname{dom} \beta$ and $x \gamma=x \beta$ for all $x \in \operatorname{dom} \gamma$. Hence $\beta=\gamma$. Therefore we deduce that $\varphi$ is a good isomorphism from $P(\operatorname{ran} \alpha)$ onto $\alpha P(\operatorname{ran} \alpha)$. This proves that $\alpha P(\operatorname{ran} \alpha) \cong P(\operatorname{ran} \alpha)$, as desired.

For any $\alpha \in E(P(X)) \backslash\{0\}$, then by Lemma 2.1. we obtain that $\alpha \circ G(X) \circ \alpha$ $=\alpha I(X) \alpha$. If we can show in Lemma 2.4., that is, if $\left|a \alpha^{-1}\right| \geq|\operatorname{ran} \alpha|$ for every $a \in \operatorname{ran} \alpha$, then $\alpha I(X) \alpha=\alpha P(\operatorname{ran} \alpha)$ and then by Lemma 2.3. we have $\alpha \circ G(X) \circ \alpha=\alpha I(X) \alpha=\alpha P(\operatorname{ran} \alpha)$ which is a subsemihypergroup of $P(X)$.

Lemma 2.4. Let $X$ be a nonempty set and $\alpha \in E(P(X)) \backslash\{0\}$. If $\left|a \alpha^{-1}\right| \geq|\operatorname{ran} \alpha|$ for every $a \in \operatorname{ran} \alpha$, then $\alpha I(X) \alpha=\alpha P(\operatorname{ran} \alpha)$.

Proof. Note that $\alpha I(X) \alpha \subseteq \alpha P(X) \alpha \subseteq \alpha P(\operatorname{ran} \alpha)$. For the reverse inclusion, let $\lambda \in P(\operatorname{ran} \alpha)$. Then $\operatorname{dom} \alpha \lambda \subseteq \operatorname{dom} \alpha$ and $\operatorname{ran} \alpha \lambda \subseteq \operatorname{ran} \lambda \subseteq \operatorname{ran} \alpha$. It follows that $c(\alpha \lambda)^{-1}=\left(c \lambda^{-1}\right) \alpha^{-1}$ for every $c \in \operatorname{ran} \alpha \lambda$ and then

$$
\operatorname{dom} \alpha \lambda=\bigcup_{c \in \operatorname{ran} \alpha \lambda} c(\alpha \lambda)^{-1}=\bigcup_{c \in \operatorname{ran} \alpha \lambda}\left(c \lambda^{-1}\right) \alpha^{-1}
$$

where $\bigcup$ stands for a disjoint union. Then

$$
\alpha \lambda=\left(\begin{array}{c}
\left(c \lambda^{-1}\right) \alpha^{-1} \\
c
\end{array}\right)_{c \in \operatorname{ran} \alpha \lambda} .
$$

We also obtain that

$$
\left|c \lambda^{-1}\right| \leq|\operatorname{ran} \alpha| \leq\left|c \alpha^{-1}\right| \text { for every } c \in \operatorname{ran} \alpha \lambda .
$$

Then for each $c \in \operatorname{ran} \alpha \lambda$, there is an injective mapping $\varphi_{c}: c \lambda^{-1} \rightarrow c \alpha^{-1}$. This implies that

$$
\left(\begin{array}{c}
x \\
x \varphi_{c}
\end{array}\right)_{\substack{c \in \operatorname{ran} \alpha \lambda \\
x \in c \lambda^{-1}}} \in I(X) .
$$

Note also that

$$
\left(\left(c \lambda^{-1}\right) \varphi_{c}\right) \alpha=\{c\} \text { for every } c \in \operatorname{ran} \alpha \lambda
$$


Thus $\alpha\left(\begin{array}{c}x \\ x \varphi_{c}\end{array}\right)_{\substack{c \in \operatorname{ran} \alpha \lambda \\ x \in c \lambda^{-1}}} \alpha \in \alpha I(X) \alpha$ and

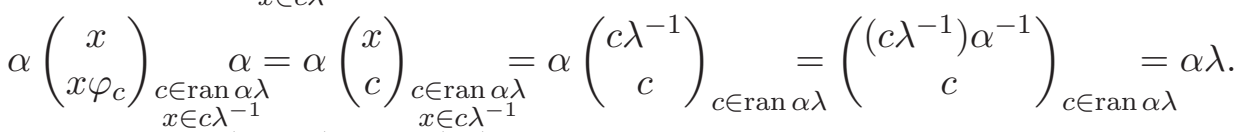

Then $\alpha P(\operatorname{ran} \alpha) \subseteq \alpha I(X) \alpha$.

Hence the proof is complete.

Next Lemma is to show that if $\alpha \circ G(X) \circ \alpha$ is a local subsemihypergroup of $P(X)$ and if $\left|a \alpha^{-1}\right|=1$ for some $a \in \operatorname{ran} \alpha$ then $\alpha$ is injective. Moreover, $\alpha \circ G(X) \circ \alpha=\alpha I(X) \alpha=I(X)$.

Lemma 2.5. Let $X$ be a nonempty set and $\alpha \in E(P(X)) \backslash\{0\}$ and assume that $\alpha \circ G(X) \circ \alpha$ is a local subsemihypergroup of $P(X)$. If $\left|a \alpha^{-1}\right|=1$ for some $a \in \operatorname{ran} \alpha$, then $\alpha$ is injective, that is, $\alpha=1_{\operatorname{dom} \alpha}$.

Proof. Assume that $\left|a \alpha^{-1}\right|=1$ for some $a \in \operatorname{ran} \alpha$. Note that $a \alpha=a$ since $a \in \operatorname{ran} \alpha$ and $\alpha \in E(P(X)) \backslash\{0\}$. Let $b \in \operatorname{ran} \alpha$ and suppose that $\left|b \alpha^{-1}\right|>1$. Let $b^{\prime} \in b \alpha^{-1}$ and $b \neq b^{\prime}$. Define $\beta, \gamma \in I(X)$ by

$$
\beta=\left(\begin{array}{cc}
a & b \\
b^{\prime} & b
\end{array}\right) \quad \text { and } \quad \gamma=\left(\begin{array}{l}
b \\
a
\end{array}\right) \text {. }
$$

Then

$$
\begin{gathered}
\alpha \beta \alpha=\left(\begin{array}{c}
x \alpha^{-1} \\
x
\end{array}\right)_{x \in \operatorname{ran} \alpha}\left(\begin{array}{cc}
a & b \\
b^{\prime} & b
\end{array}\right)\left(\begin{array}{c}
x \alpha^{-1} \\
x
\end{array}\right)_{x \in \operatorname{ran} \alpha} \\
=\left(\begin{array}{c}
x \alpha^{-1} \\
x
\end{array}\right)_{x \in \operatorname{ran} \alpha}\left(\begin{array}{ll}
a & b \\
b & b
\end{array}\right) \\
=\left(\begin{array}{c}
a \alpha^{-1} \cup b \alpha^{-1} \\
b
\end{array}\right) \in \alpha I(X) \alpha=\alpha \circ G(X) \circ \alpha \quad \text { and } \\
\alpha \gamma \alpha=\left(\begin{array}{c}
x \alpha^{-1} \\
x
\end{array}\right)_{x \in \operatorname{ran} \alpha}\left(\begin{array}{c}
b \\
a
\end{array}\right)\left(\begin{array}{c}
x \alpha^{-1} \\
x
\end{array}\right)_{x \in \operatorname{ran} \alpha}=\left(\begin{array}{c}
x \alpha^{-1} \\
x
\end{array}\right)_{x \in \operatorname{ran} \alpha}\left(\begin{array}{c}
b \\
a
\end{array}\right) \\
=\left(\begin{array}{c}
b \alpha^{-1} \\
a
\end{array}\right) \in \alpha I(X) \alpha=\alpha \circ G(X) \circ \alpha .
\end{gathered}
$$

Since $\alpha \circ G(X) \circ \alpha$ is a subsemihypergroup of $P(X)$, we have $(\alpha \beta \alpha) \circ(\alpha \gamma \alpha) \subseteq$ $\alpha \circ G(X) \circ \alpha=\alpha I(X) \alpha$. Thus $(\alpha \beta \alpha) I(X)(\alpha \gamma \alpha) \subseteq \alpha I(X) \alpha$ which implies that $(\alpha \beta \alpha)(\alpha \gamma \alpha)=\alpha \beta \alpha 1_{X}(\alpha \gamma \alpha) \in \alpha I(X) \alpha$. So,

$$
(\alpha \beta \alpha)(\alpha \gamma \alpha)=\left(\begin{array}{c}
a \alpha^{-1} \cup b \alpha^{-1} \\
a
\end{array}\right) \in \alpha I(X) \alpha .
$$


Therefore $(\alpha \beta \alpha)(\alpha \gamma \alpha)=\alpha \lambda \alpha$ for some $\lambda \in I(X)$. Thus $a(\alpha \lambda \alpha)=a \lambda \alpha=a$ and $b(\alpha \lambda \alpha)=b \lambda \alpha=a$. Hence $\{a, b\} \lambda \subseteq a \alpha^{-1}=\{a\}$ which is a contradiction since $|\{a, b\} \lambda|=2$.

This proves that for every $b \in \operatorname{ran} \alpha, b \alpha^{-1}=\{b\}$. Thus for every $x \in \operatorname{dom} \alpha$, $x \in(x \alpha) \alpha^{-1}=\{x \alpha\}$. Therefore $x \alpha=x$ for all $x \in \operatorname{dom} \alpha$, that is, $\alpha=1_{\operatorname{dom} \alpha}$, as desired.

Observe that in Lemma 2.1.- Lemma 2.4. and for any $\alpha \in E(P(X)) \backslash\{0\}$, if $\left|a \alpha^{-1}\right| \geq|\operatorname{ran} \alpha|$ for all $a \in \operatorname{ran} \alpha$ then $\alpha \circ G(X) \circ \alpha$ is a local subsemihypergroup of $P(X)$. For this reason we will show that the revers statement is also true if $X$ is a finite nonempty set.

Lemma 2.6. Let $X$ be finite nonempty set and $\alpha \in E(P(X)) \backslash\{0\}$. If $\alpha \circ G(X) \circ \alpha$ is a local subsemihypergroup of $P(X)$ and $\alpha$ is not injective, then $\left|a \alpha^{-1}\right| \geq|\operatorname{ran} \alpha|$ for all $a \in \operatorname{ran} \alpha$.

Proof. Lemma 2.5 yields that $\left|a \alpha^{-1}\right|>1$ for every $a \in \operatorname{ran} \alpha$. Thus for every $a \in \operatorname{ran} \alpha$, let $a^{\prime} \in a \alpha^{-1}$ with $a^{\prime} \neq a$.

To show that $\left|a \alpha^{-1}\right| \geq|\operatorname{ran} \alpha|$ for every $a \in \operatorname{ran} \alpha$, we are done if $|\operatorname{ran} \alpha|=$ 1. Assume that $|\operatorname{ran} \alpha|=k>1$ and let $b \in \operatorname{ran} \alpha$. Let

$$
\operatorname{ran} \alpha=\left\{b=a_{1}, a_{2}, \ldots, a_{k}\right\} .
$$

For each $i \in\{2, \ldots, k\}$, let $\beta_{i} \in I(X)$ be defined by

$$
\beta_{i}=\left(\begin{array}{ccc}
a_{1} & a_{i} & x \\
a_{1} & a_{1}^{\prime} & x
\end{array}\right)_{x \in \operatorname{ran} \alpha \backslash\left\{a_{1}, a_{i}\right\}} .
$$

Then for each $i \in\{2, \ldots, k\}$,

$$
\begin{aligned}
\alpha \beta_{i} \alpha & =\left(\begin{array}{c}
x \alpha^{-1} \\
x
\end{array}\right)_{x \in \operatorname{ran} \alpha}\left(\begin{array}{ccc}
a_{1} & a_{i} & x \\
a_{1} & a_{1}^{\prime} & x
\end{array}\right)_{x \in \operatorname{ran} \alpha \backslash\left\{a_{1}, a_{i}\right\}}\left(\begin{array}{c}
x \alpha^{-1} \\
x
\end{array}\right)_{x \in \operatorname{ran} \alpha} \\
& =\left(\begin{array}{c}
x \alpha^{-1} \\
x
\end{array}\right)_{x \in \operatorname{ran} \alpha}\left(\begin{array}{ccc}
a_{1} & a_{i} & x \\
a_{1} & a_{1} & x
\end{array}\right)_{x \in \operatorname{ran} \alpha \backslash\left\{a_{1}, a_{i}\right\}} \\
& =\left(\begin{array}{cc}
a_{1} \alpha^{-1} \cup a_{i} \alpha^{-1} & x \alpha^{-1} \\
a_{1} & x
\end{array}\right)_{x \in \operatorname{ran} \alpha \backslash\left\{a_{1}, a_{i}\right\}} .
\end{aligned}
$$

If $k=2$, then $\alpha \beta_{2} \alpha=(\operatorname{dom} \alpha)_{a_{1}}$. If $k>2$, then

$$
\left(\alpha \beta_{2} \alpha\right)\left(\alpha \beta_{3} \alpha\right)=\left(\begin{array}{cc}
a_{1} \alpha^{-1} \cup a_{2} \alpha^{-1} & x \alpha^{-1} \\
a_{1} & x
\end{array}\right)_{x \in \operatorname{ran} \alpha \backslash\left\{a_{1}, a_{2}\right\}}
$$




$$
\begin{gathered}
\left(\begin{array}{cc}
a_{1} \alpha^{-1} \cup a_{3} \alpha^{-1} & x \alpha^{-1} \\
a_{1} & x
\end{array}\right)_{x \in \operatorname{ran} \alpha \backslash\left\{a_{1}, a_{3}\right\}} \\
\quad=\left(\begin{array}{cc}
a_{1} \alpha^{-1} \cup a_{2} \alpha^{-1} \cup a_{3} \alpha^{-1} & x \alpha^{-1} \\
a_{1} & x
\end{array}\right)_{x \in \operatorname{ran} \alpha \backslash\left\{a_{1}, a_{2}, a_{3}\right\}}
\end{gathered}
$$

The following result is obtained by induction :

$$
\left(\alpha \beta_{2} \alpha\right)\left(\alpha \beta_{3} \alpha\right) \cdots\left(\alpha \beta_{k} \alpha\right)=\left(\bigcup_{i=1}^{k} a_{i} \alpha^{-1}\right)=(\operatorname{dom} \alpha)_{a_{1}} .
$$

Since $\alpha \beta_{i} \alpha \in \alpha I(X) \alpha=\alpha \circ G(X) \circ \alpha$ for each $i \in\{2, \ldots, k\}$ and $\alpha \circ G(X) \circ \alpha$ is a subsemihypergroup of $P(X)$,

$$
\left(\alpha \beta_{2} \alpha\right) \circ\left(\alpha \beta_{3} \alpha\right) \circ \cdots \circ \alpha \beta_{k} \alpha \subseteq \alpha \circ G(X) \circ \alpha=\alpha I(X) \alpha .
$$

So,

$$
\left(\alpha \beta_{2} \alpha\right) I(X)\left(\alpha \beta_{3} \alpha\right) I(X) \cdots I(X)\left(\alpha \beta_{k-1} \alpha\right) I(X)\left(\alpha \beta_{k} \alpha\right) \subseteq \alpha I(X) \alpha,
$$

which implies that

$$
\left(\alpha \beta_{2} \alpha\right)\left(\alpha \beta_{3} \alpha\right) \cdots\left(\alpha \beta_{k-1} \alpha\right)\left(\alpha \beta_{k} \alpha\right) \in \alpha I(X) \alpha
$$

We have that $(\operatorname{dom} \alpha)_{a_{1}}=\left(\alpha \beta_{2} \alpha\right)\left(\alpha \beta_{3} \alpha\right) \cdots\left(\alpha \beta_{k-1} \alpha\right)\left(\alpha \beta_{k} \alpha\right)=\alpha \gamma \alpha$ for some $\gamma \in I(X)$. Consequently,

$$
(\operatorname{dom} \alpha) \alpha \gamma \alpha=((\operatorname{ran} \alpha) \gamma) \alpha=\left\{a_{1}\right\}=\{b\}
$$

It follows that $\operatorname{ran} \alpha \subseteq \operatorname{dom} \gamma$ and $(\operatorname{ran} \alpha) \gamma \subseteq b \alpha^{-1}$. But $\gamma \in I(X)$, so we have

$$
|\operatorname{ran} \alpha|=|(\operatorname{ran} \alpha) \gamma| \leq\left|b \alpha^{-1}\right| \text {. }
$$

Hence the desired result follows.

The following theorem is obtained directly from Lemma 2.3, Lemma 2.4, Lemma 2.5 and Lemma 2.6.

Theorem 2.7. Let $X$ be finite and $\alpha \in E(P(X)) \backslash\{0\}$. Then the local subset $\alpha \circ G(X) \circ \alpha$ of $P(X)$ is a local subsemihypergroup of $P(X)$ if and only if either:

(i) $\alpha=1_{A}$ for some nonempty subset $A$ of $X$, or 
(ii) $\left|a \alpha^{-1}\right| \geq|\operatorname{ran} \alpha|$ for every $a \in \operatorname{ran} \alpha$.

Moreover,

$$
\alpha \circ G(X) \circ \alpha \begin{cases}=I(A) & \text { if } \alpha \text { satisfies (i), } \\ \cong P(\operatorname{ran} \alpha) & \text { if } \alpha \text { satisfies (ii). }\end{cases}
$$

\section{References}

[1] P.M. Higgins, Techniques of Semigroup Theory, Oxford University Press, New York (1992).

[2] P. Corsini, Prolegomena of Hypergroup Theory (second ed.), Aviani Editor, New York (1993).

[3] P. Corsini, V. Leoreanu, Applications of hyperstructor theory, Kluwer Academic Publishers (2003).

[4] P. Jampachon, Locally Factorizable Transformation Semigroups, Master Thesis, Chulalongkorn University (1984).

[5] T.W. Hungerford, Algebra, Springer-Verlag, New York (1974).

[6] P. Jampachon, M. Saicharee, R. P. Sullivan, Locally factorisable transformation semigroups, SEA Bull. Math., 25, No.2 (2001), 233-244.

[7] R.I. Sararnrakskul, S. Pianskool and Y. Kemprasit, Some Local Subsemigroups of the Full Transformation Semigroup on a Set, East-West J. Math., Spec. Vol. for ICDMA, (2008), 163-169.

[8] J. Ittharat, R.P. Sullivan, Factorisable semigroups of generalized transformations, Comm. Algebra, 33, (2005), 3179-3193.

[9] J. Ittharat, R.P. Sullivan, Factorisable semigroups of linear transformations, Comm. Alg. Colloquium, 13, No.2 (2006), 295-306. 
